\title{
Complementary use of high-resolution and high-precision cryo-ED and EM
}

Koji Yonekura $^{1,2}$, Saori Maki-Yonekura ${ }^{1}$, Kiyofumi Takaba ${ }^{1}$, Tasuku Hamaguchi ${ }^{1}$, Hisashi Naitow ${ }^{1}$ and Keisuke Kawakami ${ }^{1}$

${ }^{1}$ RIKEN SPring-8 Center, Sayo, Hyogo, Japan, ${ }^{2}$ IMRAM, Tohoku University, Sendai, Japan

Cryo-electron microscopy (cryo-EM) is widely used to determine high-resolution structures from images of protein molecules embedded in thin ice and from electron diffraction patterns of small and thin crystals. These two techniques of imaging and diffraction offer complementary structure analysis of various-sized molecules: The former single particle analysis is applied mainly to larger-sized molecules that yield good image contrast in vitreous ice; Electron 3D crystallography (3D ED / MicroED) is applicable to undersized crystals of smaller molecules, which are hard to solve by single particle analysis and do not grow to a suitable size for X-ray diffraction even with a high-intensity synchrotron radiation beam. The former application has value in structural biology, while the importance of the latter is becoming recognized in synthetic chemistry, material sciences and related areas.

Following these successes, many researchers from macromolecular X-ray crystallography (MX) and other areas have joined the cryo-EM field. Accordingly, the number of cryo-electron microscopes with a direct detection device (DDD) camera is increasing over research institutes, universities, shared facilities and industry. Most middle-range $200 \mathrm{kV}$ to high-end $300 \mathrm{kV}$ machines are equipped with a Schottky emission gun, as this gun produces a stable emission with moderate coherence during data collection over a few days. Use of a cold-field emission gun (CFEG) is still limited in cryo-EM applications despite its high temporal-coherence and superior high-resolution signals.

Likewise, use of energy filtration is rare in electron 3D crystallography. In the standard scheme, sequential frames of rotational diffraction patterns are recorded on the detector of using an electron microscope, while the sample crystal is continuously rotated as in MX. The merits of energy filtration are obvious, as thicker crystals and highly-tilted crystals yield many inelastically scattered electrons, which result in high background noise in diffraction.

We have introduced a cryo-EM system based on the 1st machine of JEOL CRYO ARM 300 equipped with a CFE gun and an in-column energy filter, and been carrying out R\&D of electron 3D crystallography and single particle analysis using this system since 2018. The former has been applied to small and thin crystals of various molecules covering proteins, polypeptides, drugs and organic materials. We have shown that combination of $300 \mathrm{kV}$ electrons and energy filtering is highly beneficial to achieve highquality structure analysis. For single particle analysis, we found that the CFE beam can enable higherresolution reconstructions with a less amplitude decay from smaller numbers of images than the conventional Schottky emission does. Our target molecules range from $\sim 50 \mathrm{kDa}$ proteins to super macromolecular complexes, which include spherical viruses, membrane proteins, protein-nucleic acids complexes, and so on. Several structures resolve individual atoms in their functional sites.

Automated and remote data collection improves throughputs in both single particle analysis and crystallography. Machine-learning approaches further accelerate these applications. In this symposium, we will report our recent results and advances by complementary use of the two technologies. 


\section{Acknowledgements}

We thank Tetsuya Ishikawa and other lab members for supporting our works, the JEOL cryo-EM group for technical assistance in setting up the CRYO ARM 300 electron microscope, Satoru Inoue, Tatsuo Hasegawa, Yasutomo Segawa, and Kenichiro Itami for collaboration on structure studies of various crystals. This work was partly supported by Japan Society for the Promotion of Science Grant-in-Aid for Challenging Exploratory Research Grant 24657111, the RIKEN Pioneering Project, Dynamic Structural Biology, the Cyclic Innovation for Clinical Empowerment (CiCLE) from the Japan Agency for Medical Research and Development, AMED, and JST CREST Grant Number JPMJCR18J2, Japan, and JST-Mirai Program Grant Number JPMJMI20G5.

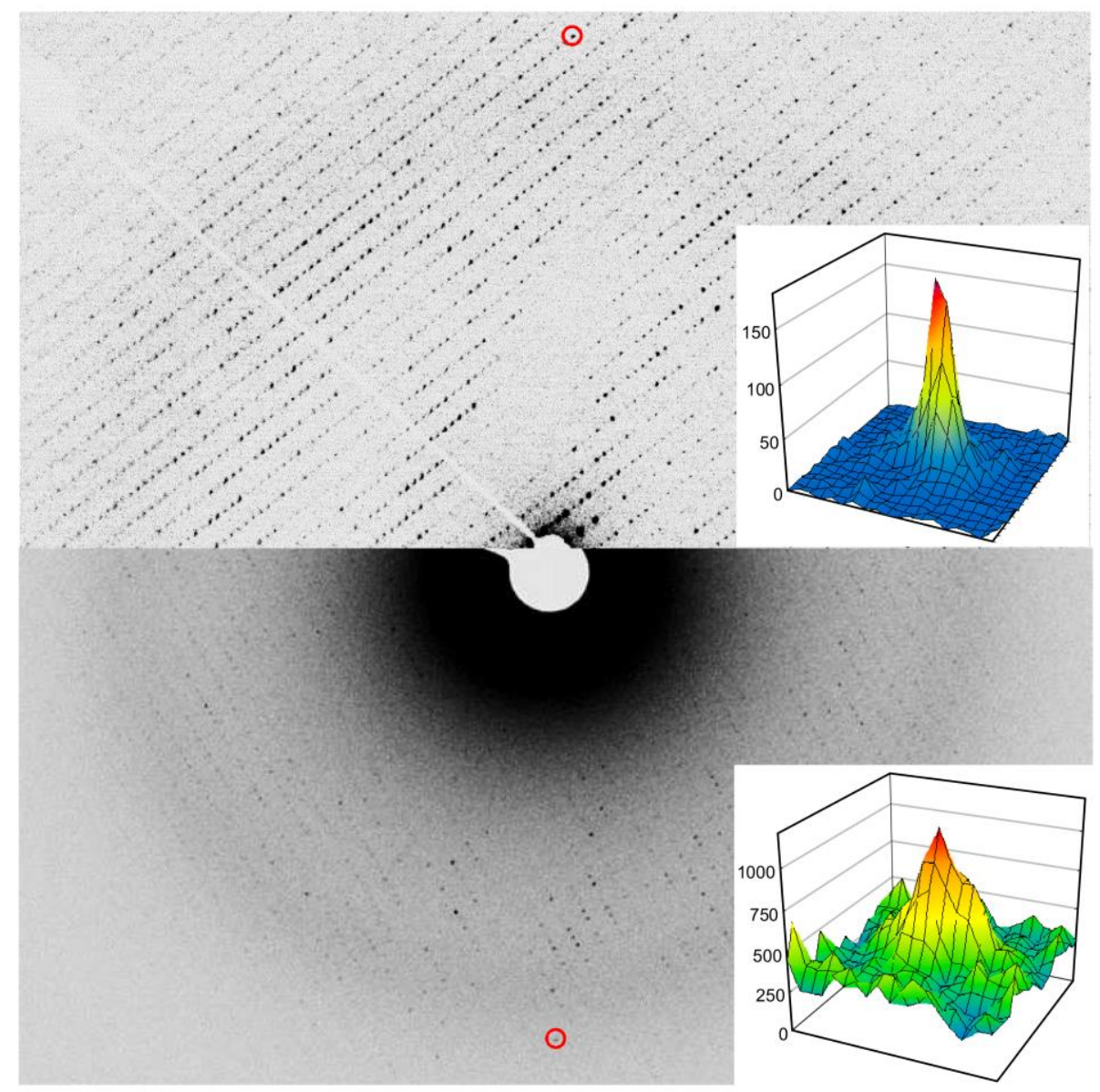

Figure 1. Electron diffraction patterns of thin 3D protein crystals. Upper: An eEFD frame taken with the CRYO ARM 300 microscope at an accelerating voltage of $300 \mathrm{kV}$ and with energy filtration. Lower: A frame taken a microscope at $200 \mathrm{kV}$ and without energy filtration. Intensity profiles of diffraction spots at $\sim 3 \AA$ (indicated by red circles) are overlaid on the diffraction patterns. 

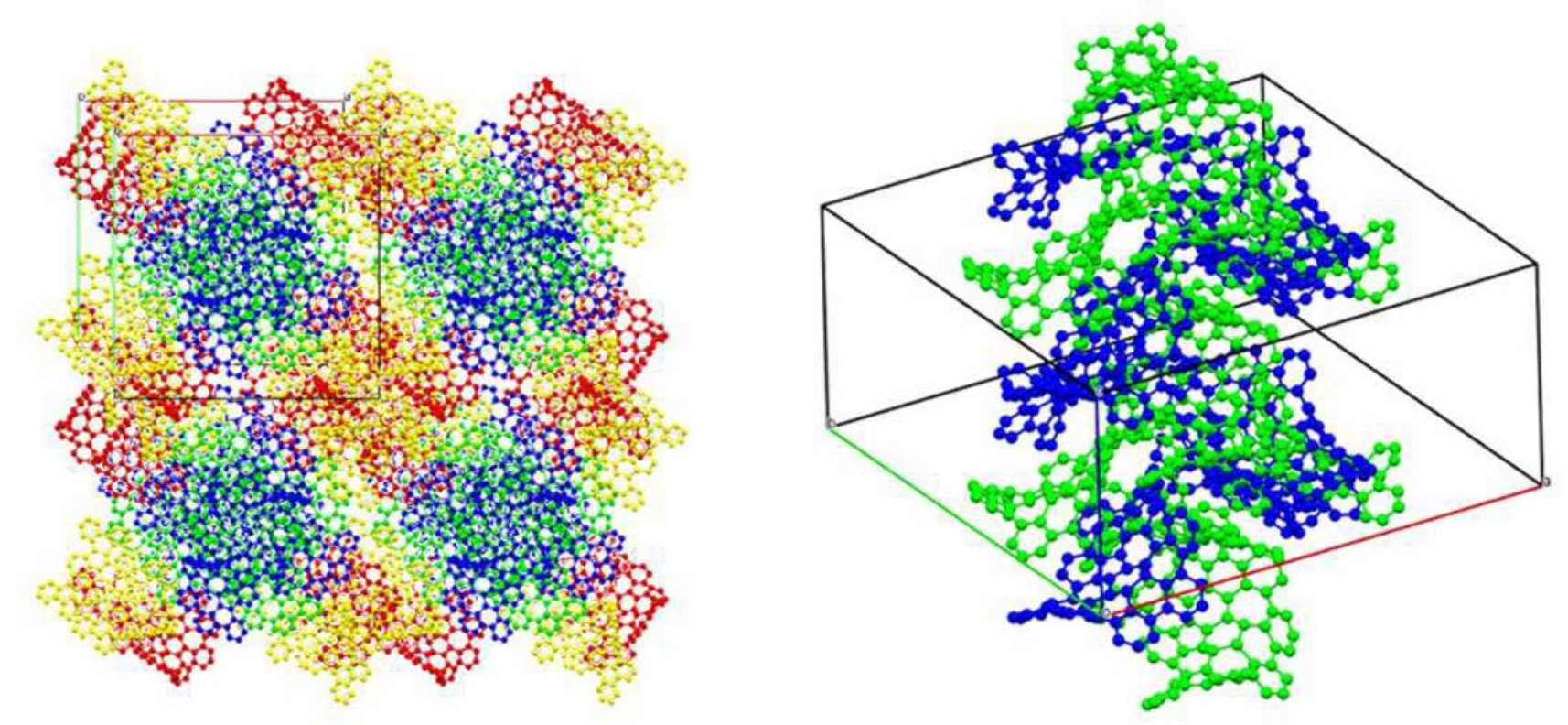

Figure 2. A nanographene double-helix fiber by eEFD.

\section{References}

Kato K., Takaba K., Maki-Yonekura S., Mitoma N., Nakanishi Y., Nishihara T., Hatakeyama T., Kawada T., Hijikata Y., Pirillo J., Scott L. T., Yonekura K., Yasutomo Segawa Y., Itami K. "Double-helix supramolecular nanofibers assembled from negatively curved nanographenes" https://chemrxiv.org/articles/preprint/Double-

Helix_Supramolecular_Nanofibers_Assembled_from_Negatively_Curved_Nanographenes/13270607 Hamaguchi T., Kawakami K., Shinzawa-Itoh K., Inoue-Kashino N., Itoh S., Ifuku K., Yamashita E., Maeda K., Yonekura K., Kashino Y., "Structure of the far-red light utilizing photosystem I of Acaryochloris" https://www.researchsquare.com/article/rs-56211/v2

Kato K., Miyazaki N., Hamaguchi T., Nakajima Y., Akita F., Yonekura K., Shen J.-R. "High-resolution cryo-EM structure of photosystem II: Effects of electron beam damage" Commun. Biol. In press.

Takaba K., Maki-Yonekura S., Inoue S., Hasegawa T., Yonekura K. "Protein and organic-molecular crystallography with 300kV electrons on an active pixel sensor" Front. Mol. Biosci. 7: 440 (2021)

Maki-Yonekura S., Hamaguchi T., Naitow H., Takaba K., Yonekura K. "Advances in cryo-EM and ED with a cold-field emission beam and energy filtration: - refinements of the CRYO ARM 300 system in RIKEN SPring-8 Center -", Microscopy doi: 10.1093/jmicro/dfaa052 (2020)

Takaba K., Maki-Yonekura S., Yonekura K. "Collecting large datasets of rotational electron diffraction with ParallEM and SerialEM” J. Struct. Biol. 211: 107549 (2020)

Guzmán-Afonso C., ..., Yonekura K., Nishiyama Y., "Understanding hydrogen bonding structure of molecular crystals by electron-and NMR-nanocrystallography”, Nat. Commun. 10: 3537 (2019)

Hamaguchi T., Maki-Yonekura S., Naitow H., Matsuura Y., Ishikawa T., Yonekura K. "A new cryo-EM system for single particle analysis", J. Struct. Biol. 207: 40-48 (2019)

Yonekura K., Ishikawa T., Maki-Yonekura S. "A new cryo-EM system for electron 3D crystallography”, J. Struct. Biol. 206: 243-253 (2019) 